\title{
Correlated response of autogeny to selection for adult starvation resistance in the blowfly, Lucilia cuprina
}

\author{
K Cooper, M Burd and KS Lefevere \\ Department of Biological Sciences, Monash University, P.O. Box 18, Melbourne, Victoria 3800, Australia
}

\begin{abstract}
We withheld sucrose from adults in three lineages of Lucilia cuprina, producing a four-fold greater mortality than in control lineages, in order to impose direct selection for carbohydrate starvation resistance. The frequency of autogeny (maturation of eggs in the absence of adult protein feeding) increased as a correlated response by an average of 4.9 to $9.2 \%$ per generation in three lineages subjected to starvation over five generations. The frequency of autogeny fluctuated but did not display a significant net change in three control lineages. Autogeny in L. cuprina seems to behave as a threshold trait, with a continuous, genetically-based underlying disposition producing discrete phenotypes. The herita-
\end{abstract}

bility of autogeny in our laboratory lineages was estimated to be 0.09 to $0.39,0.04$ to 0.36 , and -0.08 to 0.30 (95\% confidence intervals). Despite the potential for autogeny to evolve and despite protein limitation of female fecundity in Australian populations of $L$. cuprina, the trait is rare or absent in the field. Genetic variation for autogeny may be maintained, but at sub-threshold levels, by nutrient availability in the field, while trade-offs associated with autogeny probably limit the net fitness benefit of the trait and prevent the evolution of a noticeable frequency of autogenous females.

Heredity (2002) 88, 35-38. DOI: 10.1038/sj/hdy/6800005

Keywords: heritability; life history; oogenesis; threshold trait; trade-off

\section{Introduction}

Many insect species with holometabolous life cycles have evolved autogenous ovarian development, ie, the ability of adult females to mature eggs without ingestion of protein during the adult stage. Autogeny may be obligate, as in bombycid moths that lack adult mouthparts, or facultative (Chambers and Klowden, 1994). Facultative autogeny would seem to enhance fitness where adult nutritional resources are scarce or unpredictable. It also represents a major shift in life history in which a greater burden of resource acquisition is placed on the larval stage. One consequence of that burden is that autogeny may not be expressed if the larval environment, particularly larval density, does not allow sufficient uptake of resources (Baxter et al, 1973; Williams et al, 1977).

The sheep blowfly Lucilia cuprina is widely distributed throughout the semi-arid sheep grazing regions of Australia. Gravid females oviposit in warm, humid breaches on live sheep, and the larvae feed as ectoparasites, causing myiasis or 'sheep strike' that can kill the host. Autogenous females occur only very rarely in field populations (van Gerwen et al, 1987), but autogeny can evolve quickly under direct selection (restricted protein availability) in laboratory populations (Nicholson, 1957). Wild-type females in the field obtain protein meals from carcasses, dung, and the amino acid content of coccid and psyllid honeydew (Webber, 1958; Kitching, 1981). Dung

Correspondence: M Burd, Department of Biological Sciences, Monash University, P.O. Box 18, Melbourne, Victoria 3800, Australia. E-mail: martin.burd@sci.monash.edu.au

Received 23 March 2001; accepted 6 August 2001 and honeydew may not provide adequate protein (Webber, 1958), and aridity can desiccate all nutrient sources, making the protein unavailable to flies (Kitching, 1981). Vogt et al (1985) found evidence of chronic protein limitation of fecundity in L. cuprina populations in and around the Australian Capital Territory. The typical environment of sheep grazing districts in Australia would seemingly favour autogeny, at least during some seasons, so it is surprising that autogeny is rare despite its demonstrated potential to evolve in the laboratory.

In order to evolve there must be genetic variation for the trait in the wild. We suspect that autogeny in this species behaves as a polygenic threshold trait (Roff, 1996), in which a continuously distributed underlying disposition leads to binary phenotypes: autogeny above a threshold value of the underlying disposition, and anautogeny ('wild-type' in this article) otherwise. Our supposition of polygenic inheritance is reasonable because autogeny requires several behavioural or physiological alterations across developmental stages: increased rate or duration of larval feeding, increased volume of and protein density in the larval fat body, retention of protein reserves through pupation, and mobilisation of the protein reserves for yolk synthesis in adult females (Williams et al, 1977). The rarity of autogeny in field populations of L. cuprina is explicable if the autogeny threshold lies in the far upper tail of the distribution for these underlying traits.

What selective factors could maintain genetic variation for the disposition to autogeny, given that autogeny is rarely or never exposed to the direct scrutiny of selection? Autogenous female blowflies survive carbohydrate starvation longer, on average, than wild-type females, but 
have higher mortality rates than wild-type females when water is withheld (van Gerwen et al, 1987). These relationships suggests that environmental conditions such as food availability can exert indirect selection on the factors underlying autogeny. We investigated this possibility by subjecting laboratory lineages of L. cuprina to experimental carbohydrate starvation, and following any correlated changes in the frequency of autogeny. The results show the potential for such indirect selection to act on autogeny, and allow an approximate value of the heritability of autogeny to be estimated.

\section{Materials and methods}

We created six experimental lineages by interbreeding long-established laboratory strains of autogenous and wild-type Lucilia cuprina flies. Both strains were derived from the same original field population in Victoria, Australia, but had been maintained separately under laboratory conditions for over 100 generations. Flies in the autogenous strain never received protein as adults, and were therefore under strong selection for autogeny in every generation. The average level of autogeny among adult females in the autogenous strain was $85.6 \%$ (standard deviation $13.3 \%$; range $60 \%$ to $100 \% ; n=15$ cohorts). Wild-type flies were supplied with protein in advance of oviposition opportunities, and autogeny had never been observed in this strain.

We created each lineage for the experiment by placing 100 pupae from the autogenous strain and 100 from the wild-type strain in a common cage and allowing the adults to mate. Preliminary work had shown that the strains were interfertile. Interbreeding the strains enhanced genetic variation in the experimental lineages, and provided a frequency of autogeny sufficiently high to be detected without having inconveniently large populations. Each lineage was formed by an independent foundation from the stock strains. This procedure enhanced the independence of each replicate, but subjected the experiment to greater potential variation than would have been present if lineages were derived from the same foundation event. Our procedure is therefore conservative: any correlated response to selection that is consistent across lineages will have been detected in spite of extra variability from independent foundations.

The experiment was carried out in a controlled temperature room at $26^{\circ} \mathrm{C}, 60 \%$ relative humidity, and a 12 h:12 h light/dark cycle. Each lineage was held in a 27 litre cage made of wire frame and cloth mesh. Three lineages served as controls and three were assigned to a starvation treatment. Control lineages received water and sucrose ad libitum, while experimental lineages had sucrose withheld for the first 4 days after eclosion of the first adult. The average mortality imposed by this treatment was measured in eight cages at various dates throughout the experiment, and compared to the mortality rate in eight control cages.

Ovarian development was induced in both experimental and control lineages by providing $10 \mathrm{ml}$ of reconstituted blood ( $10 \%$ by weight dehydrated bovine blood in water) on each of the fifth and sixth days after eclosion of the first adult. Each 10-ml allotment would provide excess volume to our populations of about 450 adults, given that a blowfly can ingest a maximum of about 10 $\mu l$ in a single effort (Barton Browne et al, 1976). These meals removed any direct selection favouring autogeny in the experimental treatments. On the eighth day oviposition was stimulated by introducing a piece of sheep liver into the cages, from which eggs were harvested after $2 \mathrm{~h}$. Eggs were then transferred onto approximately 250 $\mathrm{g}$ of minced sheep tissue at a ratio of $0.028 \mathrm{~g}$ eggs (approximately 425 eggs) per $100 \mathrm{~g}$ meat. Pupae were eventually collected and transferred to new cages.

We measured the proportion of autogenous females in each generation of each lineage by dissecting a random sample of 50 females 9 days following eclosion. In order to carry out the dissections without compromising the experimental lineages, we created a duplicate cohort of each generation in each lineage from the same egg mass used for the primary cohort. This duplicate had protein withheld during the adult stage and was sacrificed to measure autogeny, while the primary cohort was used to continue the experiment. Individuals were considered autogenous if egg maturation had reached stage 8 (yolk occupied over $75 \%$ of the follicle) of the 10-stage classification proposed by Adams (1974) for the house fly, Musca domestica. In practice, the distinction between autogenous and wild-type females was unambiguous: females had either no yolk deposition in the absence of a protein meal or nearly complete to complete development.

The experiment was carried out over five generations. We analysed the change in the frequency of autogeny using linear regression. The heritability of autogeny and the variance of the heritability estimate for the three starvation lineages were calculated using the Crittendon-Falconer formulas (Lynch and Walsh, 1998). This method requires that we know only the proportion of autogenous individuals in a parental generation and the incidence of autogeny among their offspring. In addition to the usual assumptions of the Crittendon-Falconer formula (Lynch and Walsh, 1998), we must assume that offspring in our starvation lineages are bred from only autogenous parents. Our experimental design did not ensure this, but autogenous flies would have predominated in a parental population because they survive starvation better than wild-type flies (van Gerwen et al, 1987), and because our selection was severe (see Results). Any wild-type flies that did survive to breed would tend to lower the incidence of autogeny in the next generation and therefore reduce the parent-offspring similarity on which the heritability estimate is based. Our heritability estimates can therefore be regarded as minimum estimates.

\section{Results}

Four days of carbohydrate starvation produced an average mortality of $65 \%$ ( $n=8$ cages), compared to $16 \%$ in the control lineages ( $n=8$ cages). The difference was highly significant $\left(t_{14}=43.5, P \ll 0.001\right)$.

The frequency of autogeny in the starting generations of the six lineages varied from $9 \%$ to $29 \%$. When these starting frequencies are standardised to zero, the relative changes in subsequent generations show a contrast between starvation and control lineages (Figure 1). Based on linear regression, each starvation lineage showed a statistically significant increase in the frequency of autogeny over the five generations, with slopes ranging from $4.9 \%$ to $9.2 \%$ per generation (Table 1 ). Control lineages underwent large fluctuations (Figure 1) but with no stat- 


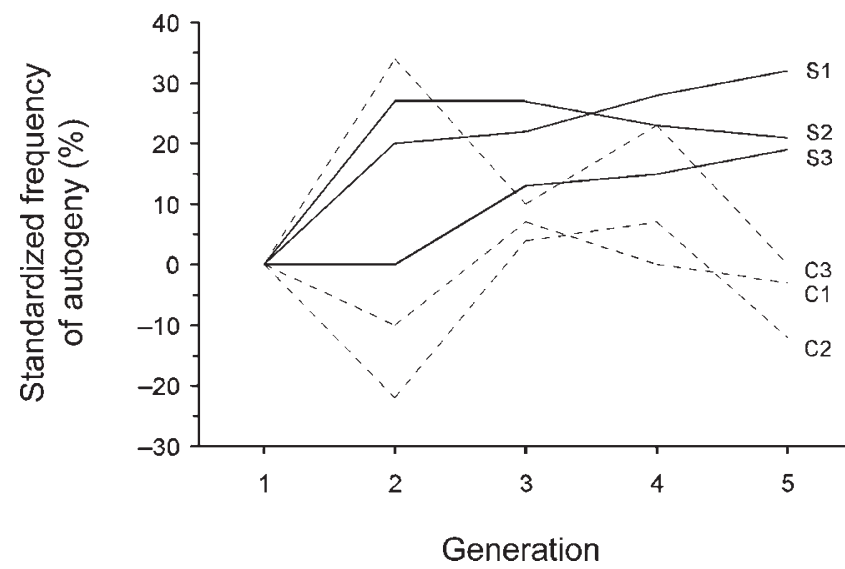

Figure 1 Frequency of autogeny in each generation of the experimental lineages. S1, S2, S3: starvation lineages; C1, C2, C3: control lineages. The ordinate values are standardised to a starting value of zero by subtracting the generation 1 frequency from all measured frequencies in that lineage. Absolute values of generation 1 frequencies are: $\mathrm{S} 1,9 \%$; S2, $12 \%$; S3, 25\%; C1, 29\%; C2, 28\%; C3, $10 \%$.

Table 1 Slopes and probability values from linear regression of standardised frequency of autogeny on generation number for each lineage

\begin{tabular}{lccc}
\hline Lineage & $\begin{array}{c}\text { Regression slope } \\
\pm 1 \text { s.e. }\end{array}$ & $t($ d.f. $=4)$ & $P$ \\
\hline Control 1 & $-0.3 \pm 1.1$ & -0.23 & 0.826 \\
Control 2 & $-1.4 \pm 2.3$ & -0.59 & 0.585 \\
Control 3 & $4.1 \pm 3.3$ & 1.25 & 0.278 \\
Starvation 1 & $9.2 \pm 1.1$ & 8.15 & 0.001 \\
Starvation 2 & $7.8 \pm 2.2$ & 3.48 & 0.025 \\
Starvation 3 & $4.9 \pm 0.5$ & 9.11 & 0.001 \\
\hline
\end{tabular}

istically significant net change over five generations (Table 1).

To calculate heritability we used generation 1 of each starvation lineage as the parental generation. The incidence of autogeny in generation 2 is subject to drift, so we estimated an average level of offspring autogeny based on the per-generation increase as determined by regression (ie, the regression slope). On this basis, heritability estimates (Lynch and Walsh, 1998, equation 25.1a) for the three starvation lineages were $0.24,0.20$, and 0.11 (Table 2). The variance of these estimates (Lynch and Walsh, 1998, equation 25.1c) yields the following 95\%

Table 2 Estimates of heritability and variance of the estimates. $A_{\mathrm{p}}$ proportion of autogenous females in generation 1

\begin{tabular}{lcccc}
\hline Lineage & $A_{\mathrm{p}}$ & $A_{\mathrm{o}}$ & $h^{2}$ & $\operatorname{var}\left(h^{2}\right)$ \\
\hline Starvation 1 & 0.09 & 0.182 & 0.24 & $6.1 \times 10^{-3}$ \\
Starvation 2 & 0.12 & 0.198 & 0.20 & $6.7 \times 10^{-3}$ \\
Starvation 3 & 0.25 & 0.299 & 0.11 & $9.6 \times 10^{-3}$ \\
\hline
\end{tabular}

$A_{\mathrm{o}}$, average proportion of autogenous females in next generation, based on per-generation rate of change determined by regression (see Table 1). $h^{2}$, Crittendon-Falconer estimate of heritability (Lynch and Walsh, 1998, equation 25.1a). $\operatorname{var}\left(h^{2}\right)$, variance of the heritability estimate (Lynch and Walsh, 1998, equation 25.1c). confidence intervals: $(0.09,0.39),(0.04,0.36)$ and $(-0.08$, $0.30)$, respectively.

\section{Discussion}

The incidence of autogeny increased under selection for starvation resistance (Figure 1), demonstrating the potential for the trait to evolve by indirect selection. The heritabilities we found have lower bounds to the confidence intervals that include or are near zero. However, these heritabilities are minimum estimates, for the reasons noted above, and the confidence intervals are commensurate with the heritabilities of many life history traits in Drosophila and other organisms (Mousseau and Roff, 1987), although lower than the average heritability for many morphological threshold traits (Roff, 1998). We cannot be certain how well these estimates apply to field populations. Populations in the wild are likely to have greater selectable variance than our laboratory lineages, even though the crossing of two laboratory strains would yield lineages with greater genetic variance than either strain individually. Given the moderate levels of heritability estimated in this study, we expect that autogeny could evolve rapidly in wild populations in response to indirect selection pressure.

It is possible that temporally variable carbohydrate availability in the adult environment is a factor maintaining genetic variation for the underlying disposition towards autogeny, even though the autogenous phenotype itself is usually absent in field populations. Autogeny and starvation resistance may share an underlying mechanism: surplus protein retained through metamorphosis may be metabolised as an energy source under starvation conditions, or used in yolk synthesis if carbohydrates are available but protein is not. Autogenous blowflies complete morphogenesis with an average of approximately $2 \mathrm{mg}$ of protein still available in the fat body and ovaries, while only about $0.6 \mathrm{mg}$ remains in the wild-type (Williams et al, 1977). Variable selection on the larval ingestion of resources and on retention of protein reserves to adulthood could yield a distribution of genotypic propensities toward autogeny, albeit a distribution rarely above the autogeny threshold.

Thus, it seems likely that, given direct or appropriate indirect selection, autogeny could evolve to high frequency in field populations of the blowfly. The rarity of autogeny implies that the trait carries trade-offs that lower the net fitness of autogenous flies in spite of their apparent reproductive advantage. We expected that such trade-offs would force autogeny to decline in the control lineages, in the absence of a selective force promoting it. In contrast, the controls appeared to be subject to drift. It seems likely that the laboratory environment was benign in comparison to the field, so that trade-offs that would otherwise be disadvantageous to autogeny had little effect.

A possible cost of autogeny would be reduced fecundity compared to wild-type females when protein is not limiting, but autogenous L. cuprina females given ad libitum access to protein achieve the same egg production as wild types (Barton Browne et al, 1981), and similarly in autogenous strains of the housefly, Musca domestica (Larsen et al, 1966).

A known cost of autogeny is greater susceptibility to desiccation (van Gerwen et al, 1987). In arid seasons when 
protein availability is most likely to limit fecundity and autogeny might otherwise be favoured, autogenous flies may suffer reduced life spans due to desiccation.

Trade-offs may also act across the life-history stages of holometabolous insects (eg, Chippendale et al, 1996). Autogeny, even in environments where it enhances adult fitness, may be associated with reduced competitive ability in the larval stage. Blowfly larvae produce considerable nitrogenous waste (Erzinçlioğlu, 1996), and autogenous larvae may ingest more waste than wild-type larvae because they must acquire greater protein reserves at this stage. In Drosophila melanogaster, increased nitrogenous waste in high density larval environments leads to the evolution of slower feeding and developmental rates (Borash et al, 2000). Similar traits for coping with or detoxifying wastes may reduce the competitive ability of autogenous larvae of L. cuprina in dense aggregations. Because L. cuprina females oviposit communally and are attracted to sites where previous oviposition has taken place (Barton Browne, 1958), a competitive larval environment is the norm.

Even long-term directional selection on threshold traits only slowly erodes genetic variance of the underlying disposition, because the unfavourable trait becomes less exposed to selection (Roff, 1994). In L. cuprina, genetic variance for autogeny is more likely to be subject to indirect selection during the larval stage (eg, selection feeding and competitive traits) or adult stage (eg, starvation resistance). It is unknown whether the rarity of expressed autogeny in natural populations is the result of inadequate indirect selection that fails to produce genotypes above the autogeny threshold, of trade-offs that limit the net fitness benefit of autogeny, or whether there might also be direct selection against autogeny.

\section{References}

Adams TS (1974). The role of juvenile hormone in housefly morphogenesis. J Insect Physiol 20: 263-276.

Barton Browne L (1958). The choice of communal oviposition sites by the Australian sheep blowfly Lucilia cuprina. Austr J Zool 6: 241-247.

Barton Browne L, Bartell RJ, van Gerwen ACM, Lawrence LA (1976). Relationship between protein ingestion and sexual receptivity in females of the Australian sheep blowfly Lucilia cuprina. Physiol Entomol 1: 235-240.

Barton Browne L, van Gerwen ACM, Williams KL (1981).
Ovarian development and oosorption in autogenous females of the blowfly. Lucilia cuprina. Int J Invert Reprod 3: 169-179.

Baxter JA, Mjeni AM, Morrison PE (1973). Expression of autogeny in relation to population density of Sarcophaga bullata Parker (Diptera: Sarcophagidae). Can J Zool 51: 1189-1193.

Borash DJ, Teotonio H, Rose MR, Mueller LD (2000). Densitydependent natural selection in Drosophila: correlations between feeding rate, development and viability. J Evol Biol 13: 181-187.

Chambers GM, Klowden MJ (1994). Nutritional reserves of autogenous and anautogenous strains of Aedes albopictus (Diptera: Culicidae). J Med Entomol 31: 554-560.

Chippindale AK, Chu TJF, Rose MR (1996). Complex trade-offs and the evolution of starvation resistance in Drosophila melanogaster. Evolution 50: 753-766.

Erzinçlioğlu Z (1996). Blowflies. Richmond Publishing: Slough, UK.

Kitching RL (1981). The sheep blowfly: a resource-limited specialist species. In: Kitching RL, Jones RE (eds). The Ecology of Pests: Some Australian Case Histories, CSIRO: Melbourne, pp 193-214.

Larsen JR, Pfadt RE, Peterson LG (1966). Olfactory and oviposition responses of the house fly to domestic manures, with notes on an autogenous strain. J Econ Entomol 59: 610-615.

Lynch M, Walsh B (1998). Genetic Analysis of Quantitative Traits. Sinauer: Sunderland, Massachusetts.

Mousseau TA, Roff DA (1987). Natural selection and the heritability of fitness components. Heredity 59: 181-197.

Nicholson AJ (1957). The self-adjustment of populations to change. Cold Spring Harbor Sympos. Quant Biol 22: 153-173.

Roff DA (1994). Evolution of dimorphic traits: effect of directional selection on heritability. Heredity 72: 36-41.

Roff DA (1996). The evolution of threshold traits in animals. $Q$ Rev Biol 71: 3-35.

Roff DA (1998). The maintenance of phenotypic and genetic variation in threshold traits by frequency-dependent selection. J Evol Biol 11: 513-529.

van Gerwen ACM, Barton Browne L, Vogt WG, Williams KL (1987). Capacity of autogenous and anautogenous females of the Australian sheep blowfly. Lucilia cuprina, to survive water and sugar deprivation following emergence. Entomol Exp Appl 43: 209-214

Vogt WG, Woodburn TL, Ellem BA, Van Gerwen ACM, Barton Browne L, Wardhaugh KG (1985). The relationship between fecundity and oocyte resorption in field populations of Lucilia cuprina. Entomol Exp Appl 39: 91-99.

Webber LG (1958). Nutrition and reproduction in the Australian sheep blowfly Luclia cuprina. Austr J Zool 3: 346-353.

Williams KL, Barton Browne L, van Gerwen ACM (1977). Ovarian development in autogenous and anautogenous Lucilia cuprina in relation to protein storage in the larval fat body. J Insect Physiol 23: 659-664. 